\title{
Von der Metaphysik- und Sprachkritik des Wiener Kreises zu Jorge Luis Borges und Ernesto Sábato
}

\author{
Strosetzki, Christoph
}

First published in:

Lateinamerika, S. 239 - 249, Vervuert, Frankfurt am Main 1999, ISBN 3-89354-114-4

Münstersches Informations- und Archivsystem multimedialer Inhalte (MIAMI)

URN: urn:nbn:de:hbz:6-02439667914 


\section{Von der Metaphysik- und Sprachkritik des Wiener Kreises zu Jorge Luis Borges und Ernesto Sábato}

CHRISTOPH STROSETZKI

"El Círculo de Viena sostuvo que la metafísica es una rama de la literatura fantástica. $Y$ ese aforismo que enfureció a los filósofos se convirtió en la plataforma literaria de Borges." (Sábato 1971: 244)

„Los mortales que hayan leído el Tractatus de Wittgenstein puieden darse con una piedra en el pecho porque es una obra difícil de conseguir: pero el Círculo de Viena se disolvió hace años, sus miembros se dispersaron sin dejar huella y yo he decidido declarar la guerra a los cavalieri della luna."(Parra 1954: 81)

Wenn man verstehen will, was Wittgenstein und der Wiener Kreis für Nicanor Parras „Advertencia al lector“ zu „Poemas y antipoemas" (1954) bedeuteten, muß man sich die Beziehung zwischen Metaphysik und Sprache vergegenwärtigen. Der Wiener Kreis führte die Probleme der Metaphysik auf die falsche Verwendung von Sprache zurück. Im "Tractatus Logico-Philosophicus" kam der frühe Wittgenstein zum vielzitierten Schluß: "Wovon man nicht sprechen kann, darüber muß man schweigen." (Wittgenstein 1960: 83) Wenn sich nun Parra als Fortsetzer einer solchen Sprachkritik sieht, dann richtet er sich jedoch nicht gegen die Tradition der abendländischen Metaphysik, sondern nach Niall Binns (1995: 83-99) gegen das als cavalieri della luna bezeichnete Dreigestirn am Himmel der chilenischen Lyrik, zu dem Pablo Neruda, Vicente Huidobro und Pablo de Rokha gehörten. So unterschiedlich die drei großen Autoren auch waren, verbindet sie doch die heftige Polemik, die sie miteinander unterhielten. Diesen drei Autoren gegenüber definiert sich Parra im selben Gedicht als Sabelius und wird damit dem Häretiker vergleichbar, der im dritten Jahrhundert das Dogma der Dreifaltigkeit umstieß. Der antidogmatische Anspruch ist es auch, den Parra bei Wittgenstein und dem Wiener Kreis bewundert.

Da Wittgensteins „Tractatus Logico-Philosophicus“ erst 1957 ins Spanische übersetzt wurde, war er im spanischen Sprachraum lange Zeit nicht verbreitet und nur wenige, wie z. B. Parra, als er sich in Oxford aufhielt, hatten das Glück, 
ihn zu lesen, während die große Mehrheit, bei der auch der Wiener Kreis keine Spur hinterlassen hatte, nach wie vor an metaphysischen Dogmen festhielt. Den antidogmatischen Anspruch des Wiener Kreises nimmt Parra für seine Antipoetik in Anspruch und lehnt damit den hermetischen und „metaphysischen“" Sprachgebrauch jener cavalieri della luna ab, die, den Blick auf den Mond gerichtet, die Realität aus den Augen verlieren, und von dem sprechen, wovon man nicht sinnvoll sprechen kann. Wie Wittgenstein war Parra Mathematiker. Während Parra in Oxford tätig war, lehrte Wittgenstein in Cambridge, wo er 1951 starb. Auch dieser biographische Hintergrund erklärt, warum Parra mit seinen in England entstandenen und 1954 erschienenen Antipoemas an Wittgenstein anknüpft. Allerdings bleibt bei Parra die Bedeutung der Metaphysik, deren Kritik er übernimmt und auf die poetologische Ebene überträgt, anders als bei Borges oder Sábato primär eine metaphorische.

\section{Der Wiener Kreis und Wittgenstein}

Worin aber besteht die Sprachkritik als Metaphysikkritik des Wiener Kreises, der Wittgenstein wichtige Anregungen zu verdanken hatte? Die Mitglieder des Wiener Kreises gaben von 1930-1938 die Zeitschrift „Erkenntnis“ heraus und mußten dann wegen des Anschlusses Österreichs an das nationalsozialistische Deutschland emigrieren. Ihr Anliegen war es, das Scheinwissen der Philosophietradition dadurch zu überwinden, daß die Logik für die Philosophie jene Rolle erhalten sollte, die die Mathematik für die Physik hatte. Der führende Kopf der Gruppe, Rudolf Carnap, rügte den im Vergleich zu den Naturwissenschaften mangelnden Erkenntnisfortschritt in der Philosophie, insbesondere in der Metaphysik. Sie solle daher künftig nicht weiter eigene Aussagen über die Welt machen, sondern die Aussagen jener Wissenschaften untersuchen, die für Aussagen über die reale Welt zuständig seien. Ziel der Philosophie solle es sein, sinnvolle von sinnlosen Sätzen zu unterscheiden. Ein Satz sei dann sinnvoll, wenn es ein Unterschied ist, ob er wahr oder falsch ist bzw. ob er verifiziert werden kann.

R. Carnap unterscheidet in seiner Schrift „Scheinprobleme in der Philoso-

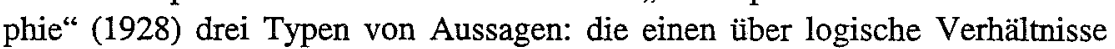
und Zusammenhänge, die anderen sind empirisch verifizierbare Sätze, und den dritten Typ bilden die Sätze, die etwas über die Wirklichkeit zu sagen beanspruchen, aber nicht verifizierbar, also sinnlos sind. Was aber sind sinnlose Aussagen? Sie sind an unterschiedlichen Merkmalen zu erkennen. So können sie, wie z.B. die „Weltseele“ oder die Eigenschaft „,babig“ (,babig" ist ein erfundenes Wort ohne Bedeutung), Wörter enthalten, die schon als solche keine klare Bedeutung haben (Camap 1966: 49). Aber auch sinnvolle Wörter können eine sinnlose Aussage bilden, wenn sie gegen die Regeln der Syntax verstoßen (z. B. sinnlose Aussage bilden, wenn sie gegen die Regeln der Syntosen Aussage sprechen, wenn sie prinzipiell nicht intersubjektiv durch Beobachtung überprüft werden kann, wobei dann auch deren Mitteilbarkeit problematisch wird. Wo man schließlich nicht klar zwischen Erkennen und Erleben unterscheidet, liegt keine beweisbare Erkenntnis vor, sondern der Ausdruck eines Erlebens, z. B. eine Gefühlslage, wie sie durch Literatur und Kunst vermittelt wird. Wer in Begriffen dichtet, ruft kein Erkennen, sondern nur ein Erleben hervor.

In „Logische Syntax der Sprache“ (1934) unterscheidet R. Cảnap, der ab 1936 in Chicago lehrt, zwischen einer Objektsprache, die sich auf die Untersuchungsgegenstände bezieht, und einer Metasprache, die sich auf die Objektsprache bezieht. Die Philosophie sollte metasprachlich ausgerichtet sein und sich auf die Objektsprache, nicht auf Objekte beziehen. Die Bemühungen um Syntax und Semantik führten ihn einerseits zur Konstruktion von Sprachen in der symbolischen Logik, andererseits zum Interesse an international konstruierten Kunstsprachen, wie dem Esperanto. Der Philosoph wird also nach Carnap zum „Konstrukteur von Sprachen“".

Ludwig Wittgenstein hatte ab 1912 in Cambridge bei Bertrand A. W. Russel studiert, der in den Naturwissenschaften das einzig mögliche Mittel der Erkenntnis sah, die Philosophie auf die Problemstellung beschränkte und ihr die Fähigkeit der Problemlösung absprach. Daraus ergab sich Wittgensteins früher positivistischer Ansatz im "Tractatus Logico-Philosophicus“, der in einer deutsch-englischen Ausgabe im Jahr 1922 erschien, und der die Metaphysik als das ablehnte, wovon man nicht sprechen kann, sondern worüber man schweigen muß. Wittgenstein selbst charakterisiert seinen Text als etwas zu Überwindendes, das man als unsinnig erkennt, wenn man es einmal verstanden hat: Der Leser ,muß sozusagen die Leiter wegwerfen, nachdem er auf ihr hinaufgestiegen iser "muß Er muß diese Sätze überwinden, dann sieht er die Welt richtig." (1960; 83) Diesen frühen Ansatz revidiert Wittgenstein in seinen ,Philosophischen Untersuchungen“ (1945). Bestehen bleibt aber die kritische Beschäftigung mit der Beziehung von Sprache und Erkenntnis. Letztere sieht er allerdings nicht mehr semantisch, sondern pragmatisch: „Ich werde auch das Ganze: der Sprache und der Tätigkeiten, mit denen sie verwoben ist, das ,Sprachspiel' nennen." (Wittgenstein 1969: 293)

Festzuhalten ist also, daß Wittgenstein und der Wiener Kreis die ungelösten Probleme der Metaphysik als Resultate falscher Sprachverwendung sehen und ihre Ausklammerung aus der Philosophie fordern, die sich künftig metasprachlich auf Sprachkritik bzw. Sprachkonstruktion zu konzentrieren habe, da Erkenntnisse über die Wirklichkeit den Naturwissenschaften vorbehalten seien. Die traditionelle Metaphysik sei nicht anders als Literatur und Kunst Ausdruck 
eines Erlebens und nicht eines Erkennens, vermittele daher keine „sinnvollen“ oder verifizierbaren Sätze. Auf diese Thesen kann man nun unterschiedlich reagieren. Man kann sie wie Sábato relativieren oder wie Borges zur Basis einer Poetik machen, in der tatsächlich die philosophische Metaphysik zum Spielball literarischen Gestaltens und Erlebens wird.

\section{Jorge Luis Borges}

Borges wurde besonders vom Philosophen und Sprachikritiker Fritz Mauthner (1849-1923) beeinflußt, der schon vor Wittgenstein davon überzeugt war, daß Sprache das wichtigste zukünftige Thema der Philosophie sei. Wie Wittgenstein betreibt er Vernunftkritik als Sprachkritik, auch wenn Wittgenstein im „Tractatus“ Russel näher steht als Mauthner. ${ }^{1}$ Silvia G. Dapía (1993) erinnert daran, daß Borges selbst gesagt hat, das „Wörterbuch der Philosophie“ Mauthners (1910) sei eines der Werke gewesen, die er immer wieder gelesen und mit Anmerkungen versehen habe (Borges 1974: 276). Arturo Echevarría konnte daher zwischen Borges und Mauthner in der Sprachauffassung drei Übereinstimmungen konstatieren: Beide sehen Sprache als willkürliches Symbolsystem, betonen den sozialen Charakter der Sprache und bestreiten die Möglichkeit der Sprache als Wirklichkeitsabbildung (Echevarría 1983; Dapía 1993: 29). Wo zum Beispiel Borges in „Tlön, Uqbar, Orbis Tertius“ Enzyklopädien anführt, will er damit die Vorstellung kritisieren, man finde darin ein vollständiges Abbild der realen Welt. ${ }^{2}$ Auch damit folgt er nach Dapía Mauthner, bei dem es angesichts der Zeitbedingtheit eines jeden Wissenssystems heißt: „Und so kam ich zu der Überzeugung: es kann ein objektives System des Wissens nicht geben, auch die äußerste Besinnung muß subjektives Menschenwerk bleiben." (1910: I, 396) Allgemeiner formuliert Mauthner: „Wissen ist Wortwissen. Wir haben nur Worte, wir wissen nichts.“ (1918: 231) Zwar sei es möglich, Ideen und Vorstellungen miteinander zu vergleichen, jedoch nie mit dem Ding an sich. Erkenntnis ist für Mauthner die Sehnsucht, über die Sprache hinauszukommen, bzw. die Illusion der Metaphysiker über die Sprache hinausgekommen zu sein (Kühn 1975: 64). Eben die Frage, ob es möglich ist, die Grenzen von Sprache, Literatur, Bewußtsein und Vorstellung zu überschreiten, um so zur

1 „4.0031- - Alle Philosophie ist ,Sprachkritik'. (Allerdings nicht im Sinne Mauthners.) Russels Verdienst ist es, gezeigt zu haben, daß die scheinbare logische Form des Satzes nicht seine wirkliche sein muB." (Wittgenstein 1969:26)

2 Es ist sicher vereinfachend, Borges' Sprachkritik wie Jaime Rest als nominalistisch zu bezeichnen, da der Nominalist in den Allgemeinbegriffen Konventionen sieht, aber die Möglichkeit der Beschreibung oder Erklärung der Welt nicht leugnet (Rest 1976: 50).
Welt an sich zu gelangen, erscheint $\mathrm{M}$. Benavides bei Borges von zentraler Bedeutung (1992: 260).

Die Beantwortung dieser Frage läßt Borges offen, indem er bewußt in seinen Texten die Linien zwischen Objektebene und Metaebene, Realität und Fiktion sowie zwischen Literatur und philosophischer Metaphysik verschwimmen läßt R. Gutiérrez Girardot formuliert es so: „Crítica literaria', ,filosofía' o, como también cabría decir, ,metafísica' e invención ,poética' no son en Borges actividades intelectuales diferentes, sino caminos del laberinto que en busca de la salida se entrecruzan,'se sorprenden recíprocamente de la busca, de sí mismos y del laberinto, pero conscientes de que el laberinto no tiene salida, siguen buscándola." (1992: 296) Insofern zieht Borges aus seiner Kritik der traditionellen Metaphysik andere Konsequenzen als der logische Positivismus. Während letzterer versuchte, eine neue, logisch exakte Sprache zu schaffen, behält Borges die Unzulänglichkeiten der metaphysischen Sprache bei und unterstreicht ihren fiktionalen Charakter, indem er sie zu Elementen von Erzählungen macht. Nicht anders ist er zu verstehen, wenn er sagt: „la metafísica es una rama de la literatura fantástica." (Borges 1974: 436)

Seine phantastischen Welten entstehen aus grammatikalischen Experimenten mit metaphysischen Implikationen. „Pensé en un mundo sin memoria, sin tiempo; consideré la posibilidad de un lenguaje que ignorara los sustantivos, un lenguaje de verbos impersonales o de indeclinables epítetos." (Borges 1974: 539) Dabei ist er als. Schriftsteller von zahlreichen Autoren vor ihm geprägt. Die Sprache übernimmt er als eine Tradition, als eine Art Weltgefühl und nicht als beliebiges Repertoire von Symbolen. (Borges 1974: 1081) Seine Erzählungen kennzeichnet ein gelehrtes Spiel mit vorhandenen oder erfundenen intertextuellen Referenzen (Blüher 1995: 119-131). Und jede einzelne Erzählung ist wiederum polyvalent und entzieht sich der eineindeutigen Zuordnung (Fleming 1993: 115-118). Besonders deutlich wird dies bei der Metapher, deren zentrale Bedeutung schon der frühe Borges des Ultraismo unterstreicht: „Hemos sinte tizado la poesía en su elemento primordial: la metáfora, a la que concedemos una máxima independencia." (Borges 1921) Deutlich wird hier auch die Vorstellung, daß Literatur primär als subjektives Erlebnis, und nicht als Aussage über die Wirklichkeit zu verstehen ist.

Nicht anders als die neuere Literaturkritik konstatierte bereits Sábato, daß Borges mit der Philosophie spielerisch und eklektizistisch umgehe. Aus ästhetischem Interesse suche er das Eigentümliche, das Unterhaltsame und Erstaunliche, wie $z$. B. logische Paradoxa, regressus ad infinitum, die er für seine Erzählungen nutze. Eklektizistisch könne Borges mal auf Parmenides, mal auf Berkeley zurückgreifen. Das sei für ihn unproblematisch, ,ya que él no se propone la verdad." (Sábato 1971: 245) Er gehe durch die Welt philosophischen Den- 
kens wie ein Sammler durch einen Antiquitätenladen. Intellektuelle Spiele mit erfundenen Welten ohne Bezug zur Realität faszinieren ihn. Philosophische oder metaphysische Erkenntnis suche er nicht. Seine Rhetorik macht ihn nach Sábato nicht zum Philosophen, sondern zum Sophisten: „quiere no participar en el siempre duro proceso de la verdad, toma del intelecto lo que tomaría un sofista: no busca la verdad sino que discute por discutir, por el solo placer mental de la discusión, y, sobre todo, eso que tanto le gusta a un literato como a un sofista: la discusión con palabras sobre palabras." (Sábato 1971: 246) Was Borges in Erzählungen wie „Tlön, Uqbar, Orbis Tertius" schreibe, können weder er noch seine Leser für wahr halten, „aunque a todos nos encanta lo que tiene de posibilidad metafísica." (Sábato 1971: 246) Und so ist es auch, wenn er von der Welt als wiederholbaren Traum, der Unsterblichkeit durch Transmigration oder durch die Erinnerung anderer spricht oder versichert, daß diese nur in der Ewigkeit existiere: „Todo es igualmente válido y nada en rigor vale.“ (Sábato 1971: 247) So kommt es, daß die Metaphysik nicht weniger Erkenntniswert hat als die phantastische Literatur. Während der Wiener Kreis zwar den Erkenntniswert der Metaphysik dem der phantastischen Literatur gleichgesetzt hat und daraus Konsequenzen für eine Reform der Philosophie zugunsten künftigen Erkenntnisgewinns ableitete, kümmerte sich Borges nicht um Abhilfe, machte aus der Not eine Tugend und schuf eine phantastische Literatur mit philosophischen Elementen. Sábato konstatiert: „El Círculo de Viena sostuvo que la metafísica es una rama de la literatura fantástica. Y ese aforismo que enfureció a los filósofos se convirtió en la plataforma literaria de Borges." (Sábato 1971: 244)

\section{Emesto Sábato}

Für Sábato dagegen geht es in der Literatur nach wie vor um Erkenntnis, gerade bei metaphysischen Fragen. ${ }^{3}$ Ihnen gegenüber erscheinen ihm die mathematischen Symbole als marmornes Museum. Das Individuum frage sich, wozu dieser Aufwand zur Beherrschung der Welt nutze sei, wenn damit ,su angustia ante los eternos enigmas de la vida y de la muerte" ungelöst bleibe. Wesentlich seien dagegen metaphysische Fragen wie „¿Tiene algún sentido la vida? ¿Qué significa la muerte? ¿Somos un alma eterna o meramente un conglomerado de

3 „Para Sábato, la narrativa es una penosa elaboración estilística tendiente a alcanzar efectos concretos (cognoscitivos); para Borges, la escritura es el resultado de una actitud de elegancia respecto a la vida. Según Sábato no se podría vivir sin literatura, sin sistemas justificativos de los pensamientos, de las opciones de todos los días; según Borges, se viviría mejor." (Campa 1983: 17) moléculas de sal y tierra? ¿Hay Dios o no?" (Sábato 1988: 61) ${ }^{4}$ Gegenüber diesen Problemen seien das Gravitationsgesetz, die Dampfmaschine und die kantischen Kategorien Kinderspiele. Wenn die Vergänglichkeit ein zentrales Problem darstelle, dann werde dies nirgendwo so betont wie in Lateinamerika. 5

Die Fragen des einzelnen Individuums können Logik und Naturwissenschaft schon deshalb nicht beantworten, weil sie sich im Allgemeinen und nicht wie die Kunst im individuell Konkreten bewege. Demgegenüber besitze der Dichter mit dem Stil ein individualisierendes Mittel. ${ }^{6}$ Die Sprache der Literatur ist in der Lage, metaphysisch zu sein und entsprechende Erlebnisse zu vermitteln. Jeder der großen Romanautoren wie Balzac, Dostojewski oder Proust, vermittelt eine „visión del mundo“, „una intuición del mundo y de la existencia del hombre; pues a la inversa del pensador puro, que nos ofrece en sus tratados un esqueleto meramente conceptual de la realidad, el poeta nos da una imagen total. [...] el artista tiene de comunicarnos una verdad sobre el cielo y el infiemo, la verdad que él advierte y sufre. No nos da una prueba, ni demuestra una tesis, ni hace propaganda de un partido o una iglesia: nos ofrece una significación." (Sábato 1971: 262) So erfolgt der dichterische Zugang zur Wahrheit nicht über den Beweis oder durch Propaganda, sondern durch ein sinnhaftes "Erleben“, wie Carnap sagen würde. Deutlich wird bei Sábatos Definition des Sinns der Kunst ex negativo auch der Einfluß des Neukantianismus: Mit Windelband stellt er der nomothetischen Naturwissenschaft die idiographische Geisteswissenschaft gegenüber, und mit Dilthey nennt er die "Weltanschauung" als Grundkategorie. Nicht durch den Neokantianismus, sondern durch die Sprachkritik des Wiener Kreises bedingt zu sein, scheint. Sábatos Frage, ob die Dichtung ähnlich wie die Naturwissenschaft eine spezifische neue Sprache benötigt. Eine Trennung jedoch von Alltagssprache und Dichtungssprache hält er für falsch, da es keine dichterischen Worte, sondern nur dichterische Sachverhalte gibt, die ihrerseits mit einer möglichst einfachen und durchsichtigen Sprache,

4 Dafür, daß das Wort Metaphysik bei einigen Interpreten Sábatos eine ebenso freie wie verschwommene Deutung erhält, ist Raúl Chavarri ein Beispiel: er unterscheidet "relaciones del hombre con cuanto le rodea", "una auténtica metafísica de la esperanza“" und ,una metafísica de lo sensible, casi próxima a las primacías de lo irracional, sobre las retóricas del raciocinio" (Chavarri 1983: 679).

5 „Pues si el problema metafísico central del hombre es su transitoriedad, aquí somos más transitorios y efímeros que en París o en Roma, vivimos como en un campamento en medio de un terremoto “ (Sábato 1971: 39).

6 „El artista es un revelador. $Y$ esa revelación se hace con una forma que se denomina estilo.“ (Sábato 1971: 207) 
d.h. mit der Alltagssprache, ausgedrückt werden sollen (Sábato 1971: 207). Dabei ist gerade die Einfachheit Resultat besonderer Anstrengung.?

Die „novela metafísica“8 hält Sábato für die angemessene Gattung. Deutlich wird bei seiner Definition der „dramas metafísicos" erneut, daß er wie Camap Metaphysik und Literatur primär als Erleben konzipiert:

„Mi narrativa se propone el examen de los dilemas últimos de la condición humana: la soledad y la muerte, la esperanza o la desesperación, el ansia de poder, la búsqueda de lo absoluto, el sentido de la existencia, la presencia o ausencia de Dios. No sé si he logrado expresar cabalmente esos dramas metafísicos, pero en todo caso es lo que me propuse. “ (Fernández 1983: 35)

Die für die Literatur wesentliche Realität ist also keine objektive, sondern eine erlebte subjektive und als solche sprachlich festgehaltene. ${ }^{9}$ Objektive Realität ohne subjektive Prägung gibt es nicht, auch nicht in der Kunst. Sábato kritisiert die poetologischen Vorstellungen der Realisten, die zu Unrecht davon ausgingen, es gebe außerhalb des Menschen eine Realität, die unabhängig von subjektiven Wahrnehmungen und Einsichten gekannt, beschrieben oder gezeichnet werden könne (Sábato 1981: 125). Bildlich veranschaulicht er seine Kritik an Stendhals Realismus:

„Suponiendo posible la reproducción fiel del mundo externo, no veo para qué esa inútil duplicación. Muchos se proponen este desatinado oficio de papel carbónico con tanta furia como ineficacia, por ignorar que el hombre es un papel carbónico que presta a la realidad externa su propio color." (Sábato 1981: 61) 10

Am Anfang und am Ende stehe das Subjekt, wie Sábato in verschiedenen Aphorismen verdeutlicht: „Uno se embarca hacia tierras lejanas, o busca el conocimiento de hombres, o indaga la naturaleza, o busca a Dios; después se advierte que el fantasma que se perseguía era Uno-mismo." (Sábato 1981: 15)11

7 „Todos los grandes escritores escriben con sencillez, pero casi siempre a costa de mucho esfuerzo." (Sábato 1971: 209)

8 Im metaphysisch geprägten Kunstwerk Sábatos erkennt Marianne Kuener Ansätze des Existentialismus, aber auch die ästhetische und philosophische Dimension wieder, die die Romantik zum Gesamtkunstwerk verbunden hatte (1991: 252f).

9 "La realidad histórica (la verdad histórica) ya no depende de los „hechos reales" acontecidos, sino que adquiere una naturaleza eminentemente verbal.“ (Femández 1983: 38)

10 Bei Sábato sind es die Figuren, die in den Romanen kämpfen, um Antworten auf ihre metaphysischen Probleme zu erlangen und das Rätsel ihrer Existenz zu entschlüsseln (Cersosimo 1992: 194; Kasner 1992: 112).

11 Sábato zitiert auch Eddington folgendermaßen: „Ha perseguido durante siglos las misteriosas huellas dejadas en la arena por alguien, hasta darse cuenta de que esas huellas eran las suyas propias." (1981: 58 )
So gibt die Literatur einer Epoche nicht objektive Sachverhalte wieder, sondern — wieder im Sinn Diltheys — subjektive „Weltanschauungen“ ihrer Zeitgenossen. ${ }^{12}$ In eine derart subjektiv geprägte Konzeption von Kunst paßt dann auch Sábatos Rückgriff auf Hegel, mit dem er den besonderen Rang der Kunst betont: „Lejos de ser simples apariencias, las formas del arte encierran más realidad y verdad que las existencias fenoménicas del mundo real." (Sábato 1971: 200)

Der Bevorzugung des Subjektiven gegenüber dem Objektiven im Leben und in der Literaturauffassung entspricht auch die Priorität des Erlebnisses gegenüber der Reflexion 13 und die Vorstellung, daß der Roman Erkenntnis als Erlebnis in einem Bereich zwischen Ideen und Leidenschaften. vermittelt. ${ }^{14}$ So lassen sich nach Sábato auch metaphysische Ängste nicht als reine İdeen vorstellen, sondern nur in Gefühle und Leidenschaften gekleidet.

„Las ideas metafísicas se convierten así en problemas psicológicos, la soledad metafísica se transforma en el aislamiento de un hombre concreto en una ciudad bien determinada, la desesperación se transforma en celos, y la novela 0 relato que estaba destinado a ilustrar aquel problema termina siendo el relato de una pasión y de un crimen." (Sábato 1971: 14)

Aus der philosophischen wird eine psychologische Metaphysik, aus dem allgemeinen Traktat ein konkreter Roman.

So sehr Sábato die Vorzüge metaphysischer Literatur hervorhebt, so sehr kritisiert er die Mängel der Naturwissenschaft. Zwar sei ihre Methode der genauen Beobachtung und der logischen Analyse durchaus von Dauer. Betrachte man jedoch ihre Erkenntnisse, muß man-feststellen, daß sie nur von zeitlich begrenzter Gültigkeit sind. So wurden die Lehren des Ptolomäus von Kopernikus überwunden. Einstein brachte eine neue Korrektur hervor, die aūch wieder durch eine komplexere Theorie korrigiert werden werde. Der naturwissenschaftliche Fortschritt lasse sich also häufig auf derartige dialektische Negationen zurückführen. (Sábato 1981: 44) Auch wenn der Laie die Macht der Naturwissenschaft bewundere und ihre Vertreter wie Albert Einstein oder Marie $\mathrm{Cu}$ rie verehre, hätten Fachvertreter bereits Zweifel daran, daß die zunehmend wachsende Abstraktion der Naturwissenschaft weiter wachsende Macht mit sich

12 „El arte de cada época trasunta una visión del mundo, la visión del mundo que tienen los hombres de esa época y, en particular, el concepto que esa época tiene de lo que es la realidad.“ (Sábato 1988: 67)

13 „Primero el hombre vive en el universo, y luego reflexiona sobre él y sobre su esencia.“ (Sábato 1971: 200)

14, ,.... [a literatura intenta una síntesis de lo racional con lo irracional, de lo subjetivo con lo objetivo." (Sábato 1971: 203) 
bringe. Dies beunruhige die Fachvertreter so sehr, daß sie daran zu zweifeln begännen, daß die Naturwissenschaft überhaupt noch in der Lage sei, die Realität zu erfassen (Sábato 1981: 26). Die gerade vom Wiener Kreis postulierte mathematisch-logische Sprache laufe Gefahr, sich zu verselbständigen und eine andere Art von phantastischer Literatur zu produzieren.

„El poder de la ciencia se adquiere gracias a una especie de pacto con el diablo: a costa de una progresiva evanescencia del mundo cotidiano. Llega a ser moriarca, pero, cuando lo logra, su reino es apenas un reino de fantasmas.“ (Sábato 1981: 27-28)

Nicht nur wegen ihrer Abstraktheit erleidet die Sprache der Naturwissenschaft einen Realitätsverlust. Sábato zitiert Russel, für den die Physik nicht deswegen mit der Mathematik gleichzusetzen ist, weil wir viel von der Außenwelt wüßten, sondern weil das, was wir von ihr wissen, sehr wenig ist. Wie könnten aber Notwendigkeit und Exaktheit als Eigenschaften der Logik und der Mathematik in der Psychologie Erfolg haben, wo sie doch schon kaum geeignet seien, die physische Realität zu erfassen, fragt Sábato (1981: 21). Gerade wegen ihrer mathematischen Form gehen der Naturwissenschaft wertvolle Bereiche der menschlichen Realität verloren:

,[...] sus emociones, sus sentimientos de arte o de justicia, su angustia frente a la muerte. Si el mundo matematizable fuera el único verdadero, no sólo sería ilusorio un palacio soñado, con sus damas, juglares y palafreneros; también lo serían los paisajes de la vigilia o la belleza de una fuga de Bach. O por lo menos sería ilusorio lo que en ellos nos emociona." (Sábato 1981: 30-31)

Die Idee des Wiener Kreises, daß die mathematisierte Sprache der Naturwissenschaften Fortschritte ermöglicht, während die Sprachen von Metaphysik und Literatur seit Jahrhunderten nicht sichtbar vorangekommen seien, nimmt Sábato auf, nicht allerdings ohne den Fortschritt zu relativieren und den Stillstand als Vielfalt gleichberechtigter Entwürfe zu deuten. „Cada época, cada pueblo encuentra el lenguaje que mejor expresa su pathos y su ethos. Y, significativamente, lo logra luchando contra la generalización del lenguaje conceptual." (Sábato 1971: 202) Die Sprache der Naturwissenschaft wie z. B. eine Theorie des Pythagoras bleibt mit ihren ausschließlich logischen Setzungen für immer den Werten von Ethik und Ästhetik fremd. Sábato stimmt dem Wiener Kreis zu, wenn er bedauert, daß es für die Naturwissenschaft von Schaden war, sich der Wörter der Alltagssprache zu bedienen, um abstrakte Sachverhalte zu symbolisieren. Da diese Wörter Konnotationen aus der Lebenswelt hatten und emotional belegt waren, mußten sie den Fortgang des Denkens behindern. So war es für Sábato eine berechtigte Konsequenz, daß man in den Naturwissenschaften „esta contaminación sentimental" dadurch beendete, daß man sich einigte auf „una tranquila multitud de símbolos desposeídos de cualquier otro significado que el convenido por sus creadores en exactos congresos internacionales." (1971: 202) Diese logische Kunstsprache ist ganz bewußt von der Alltagssprache unterschieden, die ebenso wenig logisch ist wie Alltagswelt, die nicht nur abstrakte Erkenntnisse formulieren, sondern Gefühle ausdrücken, andere beeinflussen, ihnen Sympathie oder Antipathie einflößen soll. Mit ihren Widersprüchen, Anspielungen und Absurditäten ist die Alltagssprache der Sprache der Literatur vergleichbar.

Dies zeigt sich exemplarisch in der Bedeutung der Metapher. Gerade die Tatsache, daß sie etwas anderes meint als sie bedeutet, ist ihr Vorteil. Sie ist nicht bloßer Schmuck oder rhetorische amplificatio, sondern die einzige Weise, die der Mensch hat, die subjektive Welt zum Ausdruck zu bringen. Die Metapher wird also zum paradigmatischen Ausdruck subjektiven Erlebens. Für sie gilt insbesondere, daß sie ungeeignet ist, die Wahrheiten der Logik und der Mathematik zu vermitteln, dafür aber das geeignete sprachliche Mittel für die Wahrheiten der subjektiven persönlichen Existenz mit ihren Überzeugungen, Hoffnungen und Ängsten. Obwohl die Metapher die Realität nicht abbildet, sondern Ungleiches gleichsetzt, hat sie für Sábato nicht nur einen psychologischen, sondern geradezu einen ontologischen Wert, da sie in der Lage sei, die tiefsten Schichten der Realität $\mathrm{zu}$ beleuchten. ${ }^{15}$. Mit ihrer Subjektivität und Konkretheit repräsentiert sie den äußersten Gegensatz zur mathematischlogischen Sprache der Naturwissenschaften, die zwar allgemein und objektiv, aber weltfremd und für Metaphysik und Literatur ungeeignet ist. So teilt also Sábato mit dem Wiener Kreis die Überzeugung von der Notwendigkeit einer logisch-mathematischen Sprache für die Naturwissenschaft. Er übernimmt auch die Vorstellung von der Gleichartigkeit von Literatur und Metaphysik, nicht allerdings um Metaphysik wie bei Borges zum literarischen Spiel werden zu lassen, sondern um gerade aus der Verbindung von Literatur und Metaphysik die eigentlich wichtigen Einsichten zu erwarten. Bei gleichen Prämissen ergibt sich also beim Wiener Kreis eine Aufwertung der Naturwissenschaft und eine Abwertung von Literatur und Metaphysik, während Sábato die Naturwissenschaften ab- und Literatur und Metaphysik aufwertet.

15, ,[...] tiene un valor ontológico, que actúa por alumbramiento de los estratos más profundos de la realidad." (Sábato 1981: 100) 


\section{Literatur}

Benavides, Manuel (1992): Borges y la metafísica. Cuadernos Hispanoamericanos. 505/507: 247-268.

Binns, Niall (1995): Nicanor Parra y la guerrilla literaria. Descifrando „Advertencia al lector“. Cuadernos hispanoamericanos, 537: 83-99.

Blüher, Karl Alfred (1995): Postmodernidad e intertextualidad en la obra de Jorge Luis Borges. In: Karl Alfred Blüher/Alfonso de Toro (Hrsg.): Jorge Luis Borges. Variaciones interpretativas sobre sus procedimientos literarios y bases epistemológicas. Frankfurt/Madrid, pretativas sobre sus proc

Borges, Jorge Luis (1921): Al margen de la moderna lírica. Grecia 31.

Borges, Jorge Luis (1974): Obras completas. Buenos Aires, Emecé.

Campa, Riccardo (1983): La comprensión como ficción. Cuadernos Hispanoamericanos 391/93: 7-34.

Carnap, Rudolph (1966): Scheinprobleme in der Philosophie. Frankfurt a. M., Suhrkamp.

Cersosimo, Emilse Beatriz (1992): De los carácteres a la metafísica. Revista Iberoamericana 58:193-206.

Chavarri, Raúl (1983): La metafísica y las metafísicas de Ernesto Sábato. Cuadernos Hispanoamericanos 391-93: 675-680.

Dapia, Silvia G. (1993): Die Rezeption der Sprachkritik Fritz Mauthners im Werk von Jorge Luis Borges. Köln/Weimar/Wien, Böhlau.

Echevarría, Arturo (1983): Lengua y literatura de Borges. Barcelona, Ariel.

Fernández, Teodosio (1983): Ernesto Sábato y la literatura como indagación. Cuadernos Hispanoamericanos 391/93: 35-45.

Fleming, Leonor (1993): Creación y teoría literaria a propósito de ,Las ruinas circulares’. Anthropos 142-43: 115-118.

Gutiérrez Girardot, Rafael (1992): Crítica literaria y filosofia en Jorge Luis Borges. Cuadernos Hispanoamericanos 505/507: 279-297.

Kasner, Norberto M. (1992): Metafísica y soledad: un estudio de la novelística de Ernesto Sábato. Revista Tberoamericana 58:105-113.

Kuener, Marianne (1991): Literatur und Philosophie: ihr Verhälnis bei Emesto Sábato. Frankfurt a. M.

Kühn, Joachim (1975): Gescheiterte Sprachkritik, Fritz Mauthners Leben und Werk. Berlin.

Mauthner, Fritz (1910): Wörterbuch der Philosophie. München/Leipzig, G. Müller.

Mauthner, Fritz (1918): Erinnerungen. München, G. Müller.

Parra, Nicanor (1988): Poemas y antipoemas. Madrid, Cátedra (hrsg. von René Costa)

Rest, Jaime (1976): El laberinto del universo. Borges y el pensamiento nominalista. Buenos Aires, Ediciones Librerías Fausto.

Sábato, Ernesto (1971): El escritor y sus fantasmas. Buenos Aires, Aguilar.

Sábato, Ernesto (1988): Hombres y engranajes. Heterodoxia. Madrid, Alianza Editorial.

Sábato, Emesto (1981): Uno y el universo. Edición definitiva. Barcelona/México, Seix Barral.

Wittgenstein, Ludwig (1969): Schriften. Bd. I. Frankfurt a. M., Suhrkamp. 